\title{
Special issue on selected papers from the Advanced Microelectronic and Optoelectronic Materials session of the Chinese Materials Conference 2019
}

\author{
C. Zhao ${ }^{1} \cdot$ S. N. Song ${ }^{2} \cdot$ J. Xu $^{3}$
}

Published online: 31 March 2020

(c) Springer Science+Business Media, LLC, part of Springer Nature 2020

This special issue publishes selected papers from the Session D6 of the Chinese Materials Conference 2019 (CMC2019) held in Chengdu, Sichuan Province, China, in July 10-14, 2019. CMC is the most important meeting series of the Chinese Materials Research Society (C-MRS), which is held once a year. CMC 2019, attended by more than 8000 participants, consists of 48 sessions, covering the fields of energy and environmental materials, new functional materials, high-performance structural materials, design, preparation and evaluation of materials. Session D6 was focused on advanced microelectronic and optoelectronic materials, including subjects on substrates, consumable electronic materials and thin films used for integrated circuits, optoelectronic device fabrication, new display materials, advanced electronic packaging materials, novel two-dimensional functional materials and characterization technology and methods, design theory and simulation.

All the papers published in this special issue have been selected by the guest editors based on their quality of scientific research, submitted as regular papers to this journal, and gone through the journal's regular rigorous peer-review process. The coordinating guest editors $(\mathrm{CZ})$ would like to thank the Editor-in-Chief (Professor Safa Kasap) of Journal of Materials Science: Materials in Electronics for his continuous support and the coeditors for their contribution to the completion of this special issue.

Publisher's Note Springer Nature remains neutral with regard to jurisdictional claims in published maps and institutional affiliations.
C. Zhao

zhaochao@ime.ac.cn

1 University of Chinese Academy of Sciences, Beijing, China

2 Institute of Microsystem and Information Technology, Chinese Academy of Sciences, Shanghai, China

3 Tsinghua University, Beijing, China 